\title{
Smoothing of Depinning Transitions for Directed Polymers with Quenched Disorder
}

\author{
G. Giacomin \\ Laboratoire de Probabilités de P $6 \& 7$ (CNRS U.M.R. 7599) and Université Paris 7 - Denis Diderot, \\ U.F.R. Mathématiques, Case 7012, 2 place Jussieu, 75251 Paris cedex 05, France \\ F. L. Toninelli \\ Laboratoire de Physique, ENS Lyon (CNRS U.M.R. 5672), \\ 46 Allée d'Italie, 69364 Lyon cedex 07, France
}

\begin{abstract}
We consider disordered models of pinning of directed polymers on a defect line, including $(1+1)$ dimensional interface wetting models, disordered Poland-Scheraga models of DNA denaturation and other $(1+d)$-dimensional polymers in interaction with columnar defects. We consider also random copolymers at a selective interface. These models are known to have a (de)pinning transition at some critical line in the phase diagram. In this work we prove that, as soon as disorder is present, the transition is at least of second order: the free energy is differentiable at the critical line, and the order parameter (contact fraction) vanishes continuously at the transition. On the other hand, it is known that the corresponding non-disordered models can have a first order (de)pinning transition, with a jump in the order parameter. Our results confirm predictions based on the Harris criterion.

PACS numbers: 05.70.Fh, 87.15.-v, 02.50.-r
\end{abstract}

Quenched disorder, even in arbitrarily small concentration, is expected to modify qualitatively the critical behavior of pure systems in many situations. For instance, for Ising spin systems in dimension $d \leq 2$ and for systems with continuous symmetry and $d \leq 4$ it was proven [1], via a rigorous version of the Imry-Ma argument 19, that randomness in the field conjugated to the order parameter smooths first order phase transitions. An analogous result was proven 5 for SOS effective interface models in $(2+1)$ dimensions.

In this Letter, we report on a similar phenomenon in a very different context, i.e., for $(1+d)$-dimensional directed polymer models interacting with a defect line, and for disordered copolymers at selective interfaces. Both systems are known to undergo a (de)pinning phase transition. Such models have natural applications, e.g., to biopolymers 29, 12, 26], to pinning/wetting problems 10, 11, to the problem of depinning of flux lines from columnar defects in type-II superconductors 24 and to inhomogeneous surface growth equations [21], and attracted much attention lately, both in the theoretical physics and in the mathematical literature. The pure (i.e., non-random) models present a variety of critical behaviors, ranging from first to infinite order phase transitions. On the contrary we prove that, as soon as disorder is present, the transition is always smooth. In a way, it is remarkable that one can prove such a general result on the nature of the transition, when the knowledge about the (de)pinning mechanism itself and about the location of the critical curve is still quite poor. Our result has interesting implications, in particular, on the nature of the denaturation transition for inhomogeneous Poland-Scheraga models of DNA.

Pinning/wetting models. We consider general models of directed polymers in interaction with a defect line. The seemingly abstract setting will be clarified below by some physically relevant examples. Polymer configurations are sequences $S=\left\{S_{n}\right\}_{n=0,1, \ldots}$ with values in a set $\Sigma$ which contains a specific point 0 (the origin). We set $S_{0}=$ 0 . The free polymer, in absence of interaction with the defect line, is described by a homogeneous Markov chain on $\Sigma$, with law $\mathbf{P}$. Our only assumption on $\mathbf{P}$ is the following: let $0=: \tau_{0}<\tau_{1}<\ldots$ be the return times to 0 of $S$ (of course, $\tau_{i}-\tau_{i-1}$ are independent identically distributed (IID) random variables). We require

$$
K(n) \equiv \mathbf{P}\left(\tau_{i}-\tau_{i-1}=n\right) \sim n^{-\alpha}, n \rightarrow \infty
$$

for some $1 \leq \alpha<+\infty$. Logarithmic corrections to the power decay (11) are allowed (and actually required for $\alpha=1$, to make $K(\cdot)$ summable). Note that the first return time $\tau_{1}$ has infinite mean as soon as $\alpha<2$. $S$ may be transient, i.e. $\mathbf{P}\left(\tau_{1}=\infty\right)>0$. As an example, if $S$ is the simple random walk on $\Sigma=\mathbb{Z}^{d}$, then $\alpha=3 / 2$ for $d=1$ and $\alpha=d / 2$ for $d \geq 2$. In this case, $S$ is transient as soon as $d \geq 3$. On the line $S \equiv 0$ are placed quenched IID random charges $\left\{\omega_{n}\right\}_{n=1,2, \ldots}$ of mean zero and variance one. For $\beta \geq 0$ and $h \in \mathbb{R}$, the Boltzmann distribution for the polymer of length $N$ is

$$
\mathbf{P}_{N, \omega}^{\beta, h}(S)=\mathbf{P}(S) \frac{e^{H_{N, \omega}^{\beta, h}(S)}}{Z_{N, \omega}^{\beta, h}} \mathbf{1}_{\left\{S_{N}=0\right\}},
$$

where $H_{N, \omega}^{\beta, h}(S)=\sum_{n=1}^{N}\left(\beta \omega_{n}-h\right) \mathbf{1}_{\left\{S_{n}=0\right\}}$ and of course

$$
Z_{N, \omega}^{\beta, h}=\mathbf{E}\left(e^{H_{N, \omega}^{\beta, h}(S)} \mathbf{1}_{\left\{S_{N}=0\right\}}\right),
$$

$\mathbf{E}$ denoting average with respect to $\mathbf{P}$. Note that the polymer-defect interaction takes place only at the contact points, and that a contact at $n$ with $\left(\beta \omega_{n}-h\right)>0$ is 
energetically favored. On the other hand, polymer configurations which wander away from the line are much more numerous, and therefore entropically favored, with respect to those which stay close to it. The main question is whether the interaction is enough to pin the polymer to the line. The infinite volume free energy of the model,

$$
\mathrm{F}(\beta, h)=\lim _{N \rightarrow \infty} \frac{1}{N} \log Z_{N, \omega}^{\beta, h},
$$

is self-averaging [4, 22]. Moreover, $\mathrm{F}(\beta, h) \geq 0$, as is seen restricting the partition function to the configurations which do not touch the defect line between 1 and $N-1$ : these paths have zero energy, and their entropy is not extensive, in view of (11). One then defines the pinned (or localized) region as

$$
\mathcal{L}=\{(\beta, h): \mathrm{F}(\beta, h)>0\}
$$

and the depinned (delocalized) region as

$$
\mathcal{D}=\{(\beta, h): \mathrm{F}(\beta, h)=0\} .
$$

The denominations pinned/depinned actually correspond to the typical polymer behavior. In $\mathcal{L}$ the polymer stays close to the defect line and touches it $O(N)$ times before the endpoint (various refinements of this statement are proved e.g. in 25] for a related model, the copolymer introduced below, and more recently in 17 in a more general context). On the other hand, in $\mathcal{D}$ the number of contacts is at most $O(\log N)$ 15]. The regions $\mathcal{D}$ and $\mathcal{L}$ are separated by the critical line $h_{c}(\beta)$, so that $\mathcal{D}=$ $\left\{(\beta, h): h \geq h_{c}(\beta)\right\}$.

The above model has a wide range of applications, and a vast literature is dedicated to it. Let us mention two particularly interesting examples:

- $(1+1)$-dimensional wetting of a disordered substrate [2, 10, 11]. Here, $\Sigma=\mathbb{Z}^{+}$and $\alpha=3 / 2$. The defect line represents a wall with impurities, and $S$ the interface between two coexisting phases (say, liquid below the interface and vapor above). $h<0$ means that the underlying homogeneous substrate repels the liquid phase, and vice versa for $h>0$. $\mathcal{L}$ corresponds then to the $d r y$ phase (microscopic liquid layer at the wall) and $\mathcal{D}$ to the wet phase (macroscopic layer). One of the most debated (and still unsettled) issues is whether or not the critical line coincides with that of the (exactly solvable) annealed model, where disorder is averaged in the partition function on the same footing as $S$.

- Poland-Scheraga (PS) models of DNA denaturation 9, 20]. In this case $\Sigma=\mathbb{Z}^{+}$, and $S_{n}$ represents the relative distance between two DNA strands in correspondence of the $n^{t h}$ base pair: $S_{n}=0$ if the pair is bound, $S_{n}>0$ if the bond is broken. Therefore, $\mathcal{L}$ (resp. $\mathcal{D}$ ) represents the bound (resp. denaturated) phase. Modeling $S$ as a simple random walk is known not to be physically realistic, and a phenomenological value $\alpha>2$ (loop exponent), which keeps into account the self-avoidance of the two strands, has been proposed 20]. Therefore, the transition is first order in the pure case, cf. (5) below. Of course, real DNA is intrinsically non-homogeneous and one resorts very naturally to disordered models like (2), although the IID assumption on $\omega$ is very questionable in this case.

Smoothing of the transition. The order parameter associated to the transition is the contact fraction,

$$
f_{N}=N^{-1} \mathbf{E}_{N, \omega}^{\beta, h}\left(\#\left\{1 \leq n \leq N: S_{n}=0\right\}\right)
$$

In the pure case $(\beta=0)$, critical point and critical behavior can be computed explicitly, see e.g. [2, 16]. The critical point is $h_{c}(0)=\log \left(1-\mathbf{P}\left(\tau_{1}=\infty\right)\right) \leq 0$ (notice that $h_{c}(0)<0$ iff $S$ is transient). As for the nature of the transition, one distinguishes two cases: it is of first order (the contact fraction is discontinuous in the infinite volume limit) if $\sum_{n>1} n K(n)<+\infty$, and of higher order if $\sum_{n \geq 1} n K(n)=+\infty$. In particular, if $\delta \geq 0$ then

$$
\mathrm{F}\left(0, h_{c}(0)-\delta\right) \sim \text { const } \times \delta \text { for } \alpha>2,
$$

while

$$
\mathrm{F}\left(0, h_{c}(0)-\delta\right) \sim \text { const } \times \delta^{1 /(\alpha-1)} \text { for } 1 \leq \alpha<2
$$

modulo possible logarithmic corrections. For $\alpha=1$, the transition is of infinite order.

The main result of this Letter is that, as soon as disorder is present $(\beta>0)$, the transition is always smooth:

Theorem 1 For every $\beta>0$ there exists $0<c(\beta)<$ $+\infty$ such that, for every $1 \leq \alpha<+\infty$ and $\delta \geq 0$,

$$
\mathrm{F}\left(\beta, h_{c}(\beta)-\delta\right) \leq \alpha c(\beta) \delta^{2} .
$$

Notice that, since $\mathrm{F}(\beta, h) \geq 0$, (7) is really an estimate on the regularity of the transition, an issue debated for example in the context of the disordered PS model [8, 9, 13]. In particular, (7) shows that the order of the transition is at least two, i.e., the fraction of bound base pairs vanishes continuously approaching $h_{c}(\beta)$, in contrast with the conclusions of some numerical studies [13, 14]. By convexity, self-averaging of $\mathrm{F}$ implies self-averaging of the contact fraction, whenever $\partial_{h} \mathrm{~F}(\beta, h)$ exists. Theorem 11in particular excludes the possibility of non-selfaveraging behavior of the contact fraction at the critical point, which was claimed in 13, 14]. Another interesting consequence of Theorem 1 is an upper bound on the number of pinned sites in a small window around the the critical point, for finite $N$ : indeed, one can show [28] that, if $\beta>0$ and $\left|h-h_{c}(\beta)\right| \leq$ const $\times N^{-1 / 3}$, the probability that $f_{N} \gg N^{-1 / 3}$ vanishes for $N \rightarrow \infty$. Note that, comparing (6) and (7), our result confirms Harris' criterion 18] which, translated into the present context, predicts that 
disorder is relevant and changes the nature of the transition as soon as $\alpha>3 / 2$ (it also predicts that the critical behavior does not change if $\alpha<3 / 2$, which is compatible with (7).) For previous rigorous work connected to the Harris criterion and to critical exponent inequalities for random systems, cf. [7].

As a last remark, note that Theorem 1 is reminiscent of the Aizenman-Wehr result [1] about smoothing of first order phase transitions via quenched disorder in $2 \mathrm{~d}$ spin systems (in particular, the Random Field Ising Model). However, the analogy is rather superficial and very different physical mechanisms are involved in the two cases. Indeed, 1] is based on a comparison between two competing effects: on one hand the ordering effect of boundary conditions, on the other the effect of random field fluctuations in the bulk. In our case, instead, boundary conditions play no role at all (the endpoint $S_{N}$ is pinned to 0 , cf. (2) ). Our method consists rather in selecting polymer configurations that visit rare but favorable regions with atypical disorder, and in giving Large Deviation Estimates on the number of such regions. This approach was partly inspired by [3], where a similar path selection method was used to obtain rigorous lower bounds on $\mathrm{F}(\beta, h)$ for the copolymer model.

A Large Deviations approach. Theorem[1] proven in full detail in Ref. [16], under some technical assumptions on the law of $\omega$ : the result holds in particular if $\omega_{n}$ is bounded or if it is Gaussian. Here, we present an intuitive argument which clarifies the heart of the method. Assume for simplicity a Gaussian distribution for the disorder, $\omega_{n} \sim \mathcal{N}(0,1)$. Let $1 \ll \ell \ll N$ and divide the system into $k=N / \ell$ blocks $B_{0}, \ldots, B_{k-1}$ of length $\ell$. For a given disorder realization, select the good blocks where the sum of the charges is approximately $\delta \ell$, i.e., let

$$
\mathcal{I}(\omega)=\left\{0 \leq j \leq k-1: \sum_{n=\ell j+1}^{\ell(j+1)} \omega_{n} \sim \ell \delta\right\}
$$

By elementary large deviations considerations, one realizes that there are typically $M_{t y p}=(N / \ell) e^{-\ell \delta^{2} / 2} \operatorname{good}$ blocks, two successive good blocks being separated by a typical distance $d_{t y p}=\ell e^{+\ell \delta^{2} / 2}$. Next, select all those configurations of $S$ that touch 0 at the endpoints of the good blocks $B_{j}, j \in \mathcal{I}(\omega)$ and that do not touch 0 inside the bad blocks $B_{j}, j \notin \mathcal{I}(\omega)$ (cf. Fig. 11), and call $\mathcal{S}_{\omega}$ the collection of such configurations. Of course, one obtains a lower bound on the free energy by restricting the partition sum to the selected configurations, i.e.,

$$
\frac{1}{N} \log Z_{N, \omega}^{\beta, h} \geq \frac{1}{N} \log \mathbf{E}\left(e^{H_{N, \omega}^{\beta, h}(S)} \mathbf{1}_{\left\{S \in \mathcal{S}_{\omega}\right\}} \mathbf{1}_{\left\{S_{N}=0\right\}}\right) .
$$

Thanks to the Markov property of $\mathbf{P}$, the r.h.s. of (8) factorizes into a product of terms, one for each good block and one for each excursion corresponding to a group of adjacent bad blocks (cf. Fig. 11). Note that conditioning $\ell$ independent Gaussian variables to

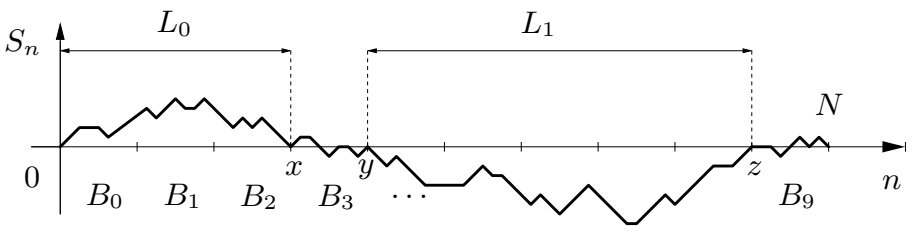

FIG. 1: A typical trajectory in $\mathcal{S}_{\omega}$. Here $k=10, \ell=8$ and $\mathcal{I}(\omega)=\{3,9\}$. Note that $S_{n} \neq 0$ for $n$ in $B_{j}$ with $j \notin \mathcal{I}(\omega)$ (bad blocks), except at the boundary with a block $B_{j}$ with $j \in \mathcal{I}(\omega)$. On the other hand, inside $B_{j}, j \in \mathcal{I}(\omega)$ (good blocks), the walk moves without constraints. The excursions $L_{0}, L_{1}, \ldots$ are typically of length $\ell \exp \left(\ell \delta^{2} / 2\right)$. The polymer is pinned to zero at steps $\{0, x, y, z, N\}$, so that $Z_{N, \omega}^{\beta, h}$ factorizes into 4 terms.

have sum $\delta \ell$ is equivalent, for $\ell$ large, to shifting the mean of each variable from 0 to $\delta$, while keeping their variance at 1 . Therefore, in each of the good blocks the polymer effectively has thermodynamical parameters $\left(\beta^{\prime}, h^{\prime}\right)=(\beta, h-\delta \beta)$. Also, note that each of the long excursions between two good blocks entails an entropic $\operatorname{loss} \log K\left(d_{\text {typ }}\right) \sim-\alpha \ell \delta^{2} / 2+O(\log \ell)$, cf. (1).

Now, take the system at the critical point, $h=h_{c}(\beta)$, and let $N \rightarrow \infty$ in (8). By the law of large numbers, the free energy contribution of good blocks converges to their density, $\rho=\ell^{-1} e^{-\ell \delta^{2} / 2}$, times the average contribution of each of them, which is $\ell\left[\mathrm{F}\left(\beta, h_{c}(\beta)-\delta \beta\right)+o(1)\right]$ for $\ell$ large (here and below the error term $o(1)$ denotes a non-random quantity that vanishes as $\ell \rightarrow \infty)$. Similarly, the contribution of excursions converges to $\rho$ times $\ell\left(-\alpha \delta^{2} / 2+o(1)\right)$. In formulas, (8) implies

$0=\mathrm{F}\left(\beta, h_{c}(\beta)\right) \geq e^{-\frac{\ell \delta^{2}}{2}}\left(\mathrm{~F}\left(\beta, h_{c}(\beta)-\delta \beta\right)-\frac{\alpha \delta^{2}}{2}+o(1)\right)$

Therefore $\mathrm{F}\left(\beta, h_{c}(\beta)-\delta \beta\right) \leq \alpha \delta^{2} / 2+o(1)$ for every finite $\ell$. Since $\ell$ is arbitrary, we obtain (7).

Copolymers at selective interfaces. Consider a polymer chain close to the interface between two solvents A and $\mathrm{B}$, and assume that some of the monomers have a larger affinity with $\mathrm{A}$ and others with $\mathrm{B}$. If the monomers are placed inhomogenously along the chain, the energetically most favored configurations will stick close to the interface. The competition with entropic effects produces also in this case a non-trivial (de)localization transition at the interface. This model was introduced in the physical literature 12, 23] and has attracted a lot of attention in the mathematical one, cf. e.g. [4, 25]. The system, although physically 3 -dimensional, can be reduced to a $(1+1)$-dimensional one if self-avoidance of the polymer is neglected [12]. Its Boltzmann distribution can be expressed, in analogy with (2), as

$$
\widehat{\mathbf{P}}_{N, \omega}^{\beta, h}(S)=\mathbf{P}(S) \frac{e^{\frac{1}{2} \sum_{n=1}^{N}\left(\beta \omega_{n}+h\right) \operatorname{sign}\left(S_{n}\right)}}{\widehat{Z}_{N, \omega}^{\beta, h}} \mathbf{1}_{\left\{S_{N}=0\right\}},
$$


with the convention $\operatorname{sign}(0)=+1$. Here, the natural setting is to take $S$ as a symmetric Markov chain on $\Sigma=\mathbb{Z}$, with increments $S_{n}-S_{n-1} \in\{-1,0,+1\}$. By symmetry, one can take $h \geq 0$. The random variables $\left(\beta \omega_{n}+h\right)$ express the affinity of the $n^{t h}$ monomer with A, and $h$ is a measure of the asymmetry of the chain (if $h>0$ there is typically a fraction $>1 / 2$ of monomers which prefer A (favorable solvent)). In the literature, the only case considered is that of symmetric random walks with IID increments $S_{n}-S_{n-1}$, which implies $\alpha=3 / 2$, but in our approach this restriction is not required and our analysis covers more general Markov processes.

Again, one introduces the free energy and, noting that in this case $\widehat{\mathrm{F}}(\beta, h) \geq h / 2$ (see e.g. [4]), one defines localized and delocalized regions $\mathcal{L}, \mathcal{D}$ according to whether strict inequality holds or not. Replica methods 29] and real-space renormalization group arguments 23] were used to attack the model, and rigorous bounds are known for $\widehat{\mathrm{F}}(\beta, h)$ and for the critical curve $h_{c}(\beta)$ separating $\mathcal{L}$ and $\mathcal{D}[3,4]$. Interestingly, recent numerical simulations plus probabilistic arguments indicate that none of the known bounds is optimal [6], which means that the (de)localization mechanism is still poorly understood.

While the physics of pinning/wetting models and of copolymers are rather different, the approach we present here is rather robust and works equally well for the two problems. Indeed, also for the copolymer model we can prove smoothness of the (de)localization transition for all $\beta>0$ and $1 \leq \alpha<\infty$ : Theorem 1 still holds, with $\mathrm{F}(\beta, h)$ replaced by $\widehat{\mathrm{F}}(\beta, h)-h / 2[16]$, so that the transition is at least second order in view of $\widehat{\mathrm{F}}(\beta, h)-h / 2 \geq 0$. Here, we give just an idea of how the heuristics above must be modified to obtain the result in this case. The main point is that (9) can be rewritten as

$$
\widehat{\mathbf{P}}_{N, \omega}^{\beta, h}(S) \propto \mathbf{P}(S) e^{-\sum_{n=1}^{N}\left(\beta \omega_{n}+h\right) \Delta_{n}} \mathbf{1}_{\left\{S_{N}=0\right\}}
$$

where $\Delta_{n}=0$ if $\operatorname{sign}\left(S_{n}\right)=+1$ and 1 otherwise. In this form, the analogy with (2) becomes more evident, the role of $\mathbf{1}_{\left\{S_{n}=0\right\}}$ being played by $\Delta_{n}$. One can again divide the system into blocks and select good ones where the sum of the charges is atypically large. However, when the selection of trajectories is performed as in (8), an extra condition has to be met: the selected trajectories, $S \in \mathcal{S}_{\omega}$, must satisfy $\Delta_{n}=0$ for $n$ in a bad block (which means $\operatorname{sign}\left(S_{n}\right)=+1$, and not just $S_{n} \neq 0$ ). Apart from that, the argument is identical as for pinning models.

Finally, note that for the copolymer the order parameter is no longer the contact fraction $f_{N}$, but rather $\widehat{f}_{N}=N^{-1} \widehat{\mathbf{E}}_{N, \omega}\left(\#\left\{1 \leq n \leq N: S_{n}<0\right\}\right)$, i.e., the fraction of monomers in the (unfavorable) solvent B, which vanishes continuously at the transition, in view of our result. Again, one can prove finite-size upper bounds of order $N^{-1 / 3}$ for $\widehat{f}_{N}$, around the critical point [28].

Conclusions. We have proved that an arbitrarily small amount of disorder is enough to smooth the (de)pinning transition in directed (co)polymer models. In particular, the transition is always at least of second order, even when it is discontinuous in the corresponding pure models. Moreover, we have given finite $N$ estimates on the order parameter at the critical point. In some literature, it is conjectured that the transition is actually of order higher than two (possibly infinite) in some situations: in particular, for the copolymer and pinning models with $\alpha=3 / 2$ 23, 27, 29]. Our result leaves this possibility open.

We thank Bernard Derrida, Thomas Garel, Massimiliano Gubinelli, Cécile Monthus and David Mukamel for fruitful discussions. Work partially supported by GIPANR project JC05_42461 (POLINTBIO).

[1] M. Aizenman and J. Wehr, Phys. Rev. Lett. 62, 2503 (1989); Commun. Math. Phys. 130, 489 (1990).

[2] K. S. Alexander and V. Sidoravicius, math.PR/0501028

[3] T. Bodineau and G. Giacomin, J. Stat. Phys. 117, 801 (2004)

[4] E. Bolthausen and F. den Hollander, Ann. Probab. 25, 1334 (1997).

[5] A. Bovier and C. Külske, J. Stat. Phys. 83, 751 (1996).

[6] F. Caravenna, G. Giacomin and M. Gubinelli, math-ph/0509065 to appear on J. Stat. Phys.

[7] J. T. Chayes, L. Chayes, D. S. Fisher and T. Spencer, Phys. Rev. Lett. 57, 2999 (1986).

[8] B. Coluzzi, cond-mat/0504080 1.

[9] D. Cule and T. Hwa, Phys. Rev. Lett. 79, 2375 (1997).

[10] B. Derrida, V. Hakim and J. Vannimenius, J. Stat. Phys. 66, 1189 (1992).

[11] G. Forgacs, J. M. Luck, Th. M. Nieuwenhuizen, and H. Orland, Phys. Rev. Lett. 57, 2184 (1986).

[12] T. Garel, D. A. Huse, S. Leibler, and H. Orland, Europhys. Lett. 8, 9 (1989).

[13] T. Garel and C. Monthus, J. Stat. Mech., Theory and Experiments, P06004 (2005).

[14] T. Garel and C. Monthus, cond-mat/0509479

[15] G. Giacomin and F. L. Toninelli, Probab. Theor. Rel. Fields 133, 464 (2005).

[16] G. Giacomin and F. L. Toninelli, math.PR/0506431 to appear on Commun. Math. Phys.

[17] G. Giacomin and F. L. Toninelli, math.PR/0510047

[18] A. B. Harris, J. Phys. C 7, 1671 (1974).

[19] Y. Imry and S.-K. Ma, Phys. Rev. Lett. 35, 1399 (1975).

[20] Y. Kafri, D. Mukamel, and L. Peliti, Phys. Rev. Lett. 85, 4988 (2000).

[21] H. Kallabis, M. Lässig, Phys. Rev. Lett. 75, 1578 (1995).

[22] N. Madras, S. G. Whittington, J. Phys. A 35, L427 (2002).

[23] C. Monthus, Eur. Phys. J. B 13, 111 (2000).

[24] D. R. Nelson and V. M. Vinokur, Phys. Rev. B 48, 13060 (1993).

[25] Ya. G. Sinai, Theory Probab. Appl. 38, 382 (1993).

[26] C. E. Soteros and S. G. Whittington, J. Phys. A: Math. Gen. 37, R279 (2004).

[27] L.-H. Tang and H. Chaté, Phys. Rev. Lett. 86, 830 (2001). 
[28] F. L. Toninelli, in preparation.

[29] A. Trovato and A. Maritan, Europhys. Lett. 46, 301 